\section{Focal liver lesions: interobserver and intraobserver agreement of three-dimensional contrast-enhanced ultrasound-assisted volume measurements}

\author{
Tommaso Vincenzo Bartolotta ${ }^{1,2}$, Antonio Sidoti Pinto ${ }^{1}$, Roberto Cannella1, \\ Giorgia Porrello ${ }^{1}$, Rossana Taravella ${ }^{1}$, Angelo Randazzo ${ }^{1}$, Adele Taibbi ${ }^{1}$ \\ ${ }^{1}$ Section of Radiology - BiND, University Hospital "Paolo Giaccone", Palermo; 'Department \\ of Radiology, Fondazione Istituto Giuseppe Giglio, Palermo, Italy
}

Purpose: This study was conducted to assess the interobserver and intraobserver agreement of three-dimensional contrast-enhanced ultrasound (3D-CEUS) volume calculations of focal liver lesions (FLLS).

Methods: Thirty-nine patients ( 15 men and 24 women; mean age, 55.4 years) with 39 FLLs (mean size, $3.1 \pm 1.8 \mathrm{~cm}$; size range, 1 to $8 \mathrm{~cm}$ ) prospectively underwent 3D-CEUS. Four readers calculated the volume of each lesion in an independent and blinded fashion in two separate sessions by means of a semi-automatic, commercially available proprietary software. The differences in lesion volumes $\left(\mathrm{cm}^{3}\right)$ among sessions and readers were assessed using the MannWhitney $\mathrm{U}$ test and the Kruskal-Wallis test. Bland-Altman analysis was also performed. Intraclass correlation coefficients (ICCS) with 95\% confidence intervals (95\% Cls) were calculated. The statistical significance level was set at $\mathrm{P}<0.05$.

Results: Among readers, there were no statistically significant differences in the first $(P=0.953)$ and second $(P=0.592)$ reading sessions for volume calculations of the $39 \mathrm{FLLS}$, with almost perfect inter-reader agreement (ICC values of the first reading session, $0.996 ; 95 \% \mathrm{Cl}, 0.992$ to 0.998 and ICC value of the second reading session, $0.994 ; 95 \% \mathrm{Cl}, 0.990$ to 0.997 , respectively). For each of the four readers, there were no significant differences in volume calculations between the two sessions ( $P=0.503-0.924)$, and the intrareader agreement was almost perfect for each reader (R1: ICC, $0.995 ; 95 \% \mathrm{Cl}, 0.991$ to $0.998 ; \mathrm{R} 2: \mathrm{ICC}, 0.995 ; 95 \% \mathrm{Cl}$, 0.988 to 0.997 ; R3: ICC, $0.996 ; 95 \% \mathrm{Cl}, 0.992$ to 0.998 ; R4: ICC, $0.985 ; 95 \% \mathrm{Cl}, 0.971$ to 0.992 ). Conclusion: 3D-CEUS volume calculations provided consistent measurements across different readers with almost perfect intrareader agreement.

Keywords: 3D ultrasound; Liver diseases; Contrast-enhanced ultrasonography; Tumor volume

e-ultrasonography.org

Ultrasonography 40(3), July 2021

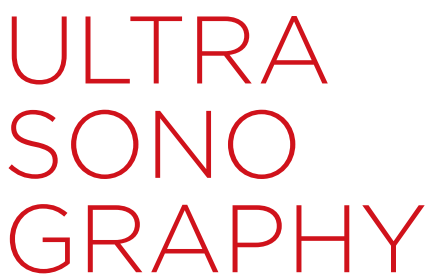

ORIGINAL ARTICLE

https://doi.org/10.14366/usg.20025 pISSN: 2288-5919 • elSSN: 2288-5943 Ultrasonography 2021;40:333-341

Received: February 14, 2020

Revised: August 18, 2020

Accepted: August 19, 2020

Correspondence to:

Roberto Cannella, MD, Section of Radiology - BiND, University Hospital

"Paolo Giaccone", Via del Vespro 129, 90127 Palermo, Italy

Tel. +39-91-6552330

E-mail: rob.cannella89@gmail.com

Tommaso Vincenzo Bartolotta, MD, PhD, Section of Radiology - BiND, University Hospital "Paolo Giaccone", Via del Vespro 129, 90127 Palermo, Italy

Tel. +39-91-6552330

Fax. +39-91-6552337

E-mail: tommasovincenzo.bartolotta@ unipa.it

This is an Open Access article distributed under the terms of the Creative Commons Attribution NonCommercial License (http://creativecommons.org/ licenses/by-nc/4.0// which permits unrestricted noncommercial use, distribution, and reproduction in any medium, provided the original work is properly cited.

Copyright (? 2021 Korean Society of Ultrasound in Medicine (KSUM)

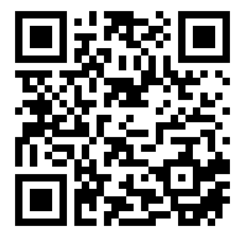

How to cite this article:

Bartolotta TV, Sidoti Pinto A, Cannella R, Porrello G, Taravella R, Randazzo A, et al. Focal liver lesions: interobserver and intraobserver agreement of three-dimensional contrastenhanced ultrasound-assisted volume measurements. Ultrasonography. 2021 Jul:40(3):333-341. 


\section{Introduction}

Contrast-enhanced ultrasonography (CEUS) is a safe, robust, and cost-effective imaging modality for evaluating focal liver lesions (FLLs) by depicting tumor vascularity in real time [1-3].

Nevertheless, an inherent limitation of conventional twodimensional CEUS (2D-CEUS) is its ability to visualize only the scanning plane, making it impossible to evaluate the vascularity of the entire lesion unless the ultrasound beam is swept across the lesion. In this regard, three-dimensional CEUS (3D-CEUS) has been shown to improve the evaluation of tumor vascularity in comparison to conventional 2D-CEUS [4-6]. Computed tomography (CT) can also display the spatial structure of FLLS, but 3D-CEUS makes it possible to evaluate the response repeatedly during shortterm follow-up, without the use of ionizing radiation or potentially nephrotoxic contrast agents $[7,8]$.

In particular, the dataset acquired by 3D-CEUS may be further processed by means of semi-automatic software, thereby allowing volume calculation and three-dimensional rendering, which have been demonstrated to improve the diagnosis, therapeutic monitoring, and follow-up of patients with liver cancer $[9,10]$. However, there is a lack of evidence about the reproducibility of 3D-CEUS volume calculations.

We undertook this study to investigate interobserver and intraobserver variability in volume calculations of FLLs by means of 3D-CEUS.

\section{Materials and Methods}

\section{Patient Population}

Institutional review board approval was obtained and full written informed consent was obtained for this prospective study (IRB \#4/2019). Our study complied with the terms of the Declaration of Helsinki.

From February 2019 to August 2019, 39 patients (24 women and 15 men; mean age, $55.4 \pm 16$ years; age range, 20 to 79 years) with $39 \mathrm{FLLs}$ (mean size at baseline ultrasound, $3.1 \pm 1.8 \mathrm{~cm}$; size range, 1 to $8 \mathrm{~cm}$ ) prospectively underwent 3D-CEUS.

The inclusion criteria for the present study were as follows: (1) at least one FLL detectable at baseline ultrasound (in case of multiple lesions, the most suitable lesion for CEUS was chosen), and (2) a lesion depth no more than $12 \mathrm{~cm}$. The exclusion criteria were as follows: (1) any major contraindication to ultrasound contrast agent administration (right-to-left shunt, pulmonary hypertension, or unstable cardiopulmonary conditions) and (2) lack of a reference standard for the final diagnosis.

Thirty of the 39 patients (77\%) underwent CEUS for the characterization of a FLL detected but undetermined at baseline ultrasonography (US), whereas the remaining nine patients (23\%) underwent CEUS for the assessment of a hepatocellular carcinoma (HCC) nodule 1 month after radiofrequency ablation (RFA).

\section{D-CEUS}

Two experienced radiologists (with more than 10 years of experience in CEUS for liver imaging), who were aware of the patients' clinical histories, performed US scanning by means of an iU22 unit (Philips Ultrasound, Bothell, WA, USA), provided with a (5-1 MHz convex array probe and Pulse Inversion imaging software. $A$ baseline survey examination, including a color/power Doppler examination and spectral analysis, was performed. Once set, the US scan parameters, such as focal zone and time gain compensation, were not changed throughout the study. The ultrasound contrast agent used in the present study was sulfur hexafluoride (SonoVue, Bracco Imaging, Milan, Italy), which was injected intravenously as a $2.4-\mathrm{mL}$ bolus followed by a $10-\mathrm{mL}$ normal sterile saline flush using a 20 - or 22-gauge peripheral intravenous cannula. A low frame rate $(5 \mathrm{~Hz})$ and a very low mechanical index (MI), ranging from 0.05 to 0.08 , were used for real-time imaging. One focus was positioned below the level of the lesion.

Digital cineloops were registered during both baseline and postcontrast US in the arterial, hepatic venous, and extended hepatic venous or late phases (i.e., 5-40 seconds, 55-90 seconds and until 200-300 seconds from the beginning of the injection, respectively).

\section{D-CEUS}

In the same session, with an interval at least of 15 minutes to allow for clearance of the previous contrast injection, the same radiologist who performed baseline ultrasound and 2D-CEUS also performed 3D-CEUS scanning using the same iU22 unit provided with a volumetric mechanical V6-2 $\mathrm{MHz}$ probe and Pulse Inversion imaging software. The specific inclusion criterion for 3D-CEUS was an FLL depth no more than $12 \mathrm{~cm}$. Harmonic imaging was used and the image was optimized for size, tissue contrast, and resolution. Depending on the size and depth of each lesion, an appropriate sweep angle (angle range, $40^{\circ}$ to $55^{\circ}$ ) was selected in order to encompass the entire lesion. SonoVue (Bracco Imaging) was then injected intravenously as a $4.8-\mathrm{mL}$ bolus followed by a $10-\mathrm{mL}$ normal sterile saline flush. For 3D-CEUS, a full bolus of $4.8 \mathrm{~mL}$ of sulfur hexafluoride was injected in order to compensate for some of the technical advantages of the $\mathrm{C} 5-1 \mathrm{MHz}$ probe over the $\mathrm{V} 6-2$ $\mathrm{MHz}$ probe, such as its operating frequency and PureWave crystals, which provide a better nominal penetration power for the C5-1 $\mathrm{MHz}$ probe. With suspended respiration, the 3D sweep was started and three volumetric datasets were acquired in the arterial, hepatic 
venous, and extended hepatic venous or late phases (i.e., $25-40$ seconds, 60-90 seconds, and 200-300 seconds from the beginning of the injection, respectively). A very low $\mathrm{MI}$, ranging from 0.06 to 0.08 , was used and each volume acquisition lasted 2-4 seconds depending on the selected sweep angle, with a longer acquisition time for greater angles. Each examination lasted for about 5 minutes after the bolus injection.

All images and cineloops were digitally stored both as raw data in a PC-based workstation connected to the US units via a standard Ethernet link and sent to our picture archiving and communication system (Impax, Agfa-Gevaert, Milan, Italy).

\section{Image Analysis}

Four readers (three second-year radiology residents and one final-year radiology senior resident) independently and randomly reviewed the 3D-CEUS examinations off-line. The readers were not involved in the scanning and were blinded to the final diagnosis, as well as to the identity, clinical history, and any other imaging findings of the patients. Two consecutive interpretation sessions with a 7-day interval to avoid recall bias were held to complete the review process. First, the readers reviewed the entire exam and chose the best phase (i.e., arterial, hepatic venous, or late phase) to calculate lesion volume. The 3D volumes were calculated using a semi-automatic, commercially available proprietary software (QLAB, Philips Ultrasound) approved for clinical use and equipped with a volume rendering mode. This mode provides the capability of displaying the data set in three orthogonal planes. In order to better display the region of interest, readers were able to freely rotate and zoom the volume, as well as to choose the appropriate depth and adjust the degree of contrast. After defining and zooming in on the area of interest, the reader manually drew a line along the long axis of the lesion and the software automatically sectioned the lesion perpendicularly to the defined long axis, providing 15 slices (distance between the individual slice, 0.3 to $5.5 \mathrm{~mm}$ ). Then the reader manually contoured the lesion slice by slice and when the latest contour was completed, the system automatically calculated the lesion volume in milliliters, also providing a 3D rendering.

For each lesion, the readers were asked to report the calculated volume and the time needed for a complete evaluation of each single 3D volumetric dataset, including defining, orienting, and zooming in on the area of interest. The QLAB software provides volume measurements in milliliters, while the International System of Units uses cubic centimeters. We converted the obtained values into cubic centimeters using the simple formula $1 \mathrm{~mL}=1 \mathrm{~cm}^{3}$. One week before starting the image analysis process, all the readers involved in this study attended a separate 5 -hour training session and all readers had to evaluate 10 other FLLs (encompassing both benign and malignant masses, which were not included in the final study) individually and separately using QLAB.

\section{Reference Standard}

The final diagnosis was obtained by magnetic resonance imaging (MRI) with hepatocellular-specific gadolinium-based contrast agent in all 39 patients. MRI scans were performed on the same day as 3D-CEUS. Follow-up by means of both CT and/or MRI, laboratory data, and clinical assessments were also used to confirm the diagnosis. MRI was performed with a 1.5-T unit (Signa Excite HDXT, General Electric Healthcare, Milwaukee, WI, USA). The MRI protocol included pre-contrast axial breath-hold and respiratory-triggered T2-weighted fast spin echo sequences with and without fatsaturation, as well as unenhanced (in-phase and out-of-phase) T1weighted and pre-contrast fat-saturated spoiled 3D gradient echo T1-weighted sequences. A triphasic dynamic contrast-enhanced study was obtained after the administration of an IV bolus of 0.1 $\mathrm{mmol} / \mathrm{kg}$ of gadobenate dimeglumine (MultiHance, Bracco Imaging). The dynamic study was followed by a hepatocellular-specific phase obtained 2 hours after the injection of contrast material.

\section{Statistical Analysis}

Data were summarized as mean and standard deviation for continuous variables and as numbers and percentages for categorical variables. Differences in categorical variables were assessed using the Pearson chi-square or Fisher exact test. The differences in lesion volumes $\left(\mathrm{cm}^{3}\right)$ in the two sessions and among readers were assessed using the Mann-Whitney $U$ test and the Kruskal-Wallis test, respectively. Intraclass correlation coefficients (ICCS) with 95\% confidence intervals (Cls) was calculated to assess the intrareader and inter-reader reliability among the four different readers, overall and for each lesion type. Inter-reader agreement was also evaluated according to the postcontrast phase, lesion localization, and size.

Agreement was categorized as poor (ICC, $<0.00)$, slight (ICC, 0.00-0.20), fair (ICC, 0.21-0.40), moderate (ICC, 0.41-0.60), substantial (ICC, $0.61-0.80)$, or almost perfect (ICC, $0.81-1.00)$.

Bland-Altman analysis was performed to evaluate the magnitude to variation between readers and the two reading sessions. The averages of the differences and $95 \%$ limits of agreement $(95 \%$ LOAs) were calculated.

The statistical significance level was set at $P<0.05$. The statistical analysis was conducted using SPSS version 20.0 (IBM Corp., Armonk, NY, USA) and MedCalc version 14.8 (MedCalc Software, Ostend, Belgium). 


\section{Results}

No adverse events were observed in our patients during or immediately after the injection of contrast agent. None of the 3DCEUS examinations were considered inadequate for volumetric analysis.

According to the reference standard, 17 of the 39 FLLS (44\%) were benign and $22 \mathrm{FLLs}(56 \%)$ were malignant. Among the 17 benign lesions, one (3\%) was hepatocellular adenoma (HCA), nine $(23 \%)$ were focal nodular hyperplasia, six $(15 \%)$ were hemangiomas, and one (3\%) was a regenerative nodule. Among the 22 malignant lesions, six (15\%) were HCC nodules, nine (23\%) lesions were HCC nodules after RFA treatment, and seven (18\%) were liver metastases (three from breast cancer, two from colon cancer, one from a gastrointestinal tumor, and one from gastric cancer).

Ten lesions were located in liver segment VI (25\%), eight (20\%) in segment VII, seven (18\%) in segment IV, seven (18\%) in segment $\mathrm{V}$, five $(13 \%)$ in segment VIII, one (3\%) in segment III, and one (3\%) in segment II.

Table 1 shows overall the intrareader and inter-reader agreement of the volume calculations. Overall, the calculated mean volume was $26.6 \pm 40.1 \mathrm{~cm}^{3}$ (volume range: $0.5-180 \mathrm{~cm}^{3}$ ), without significant differences (first session, $\mathrm{P}=0.953$; second session, $\mathrm{P}=0.592$ ) among different readers. The observed ICC for inter-reader agreement was $0.996(95 \% \mathrm{Cl}, 0.992$ to 0.998$)$ for the first session and 0.994 (95\% $\mathrm{Cl}, 0.990$ to 0.997$)$ for the second session. For each of the four readers, there were no significant differences in volume calculations between the two session $(P=0.503-0.924)$, while the intra-reader agreement ranged from 0.985 to 0.996 . Bland-Altman plots showed that the mean difference between inter-reader measurements ranged from to $-0.2 \mathrm{~cm}^{3}\left(95 \% \mathrm{LOA}, 16.4\right.$ to $\left.-16.7 \mathrm{~cm}^{3}\right)$ to -3.0 $\mathrm{cm}^{3}\left(95 \%\right.$ LOA, 12.9 to $\left.-18.9 \mathrm{~cm}^{3}\right)$ (Fig. 1). Specifically, the mean differences between the two sessions were $1.3,-2.5,-0.6$, and 2.3 $\mathrm{cm}^{3}$ for readers $1,2,3$, and 4 , respectively (Fig. 2).

Tables 2 and 3 present details on volume calculations grouped according to lesion type (Fig. 3), with inter-reader and intrareader agreement, respectively. The observed ICC ranged from 0.976 to 0.999, corresponding to almost perfect inter-reader and intrareader agreement. Almost perfect inter-reader agreement was observed regardless of the postcontrast phase chosen for volume assessment (Table 4). For volume assessment, the four readers independently chose the arterial phase for 17 of the 39 FLLs (44\%), the portalvenous phase for nine FLLs (23\%), and the late phase for 13 FLLs $(33 \%)$. A statistically significant tendency was observed for the lesions to be measured in the arterial phase $(\mathrm{P}=0.013)$.

The complete evaluation of a single 3D volumetric dataset, including defining and zooming in on the area of interest, contouring the lesion, and calculating the volume lasted on average $118 \pm 25.5$ seconds (range, 75 to 150 seconds).

\section{Discussion}

Since its introduction in clinical settings, 3D US has been widely used in gynecology, obstetrics, and cardiology, and in recent years, its use in liver imaging has also gained interest [11]. Nevertheless, volume rendering techniques cast "rays" through the 3D voxel-based volume and project the resulting image onto a $2 \mathrm{D}$ plane. In this situation, suboptimal results are obtained when there is not enough contrast between the examined structure and the adjacent tissues, such as in liver parenchyma [11]. The injection of microbubble-based contrast agents allows 3D-CEUS to overcome this limitation, paving the way for new applications of ultrasonography of the liver [4-6].

The availability of dedicated software for 3D-CEUS, such as $Q L A B$, allows a precise assessment of the shape and volume of a liver mass $[9,10]$. In comparison with CT measurements of liver

Table 1. Overall intrareader and inter-reader agreement of volume calculations of 39 focal liver lesions in two different reading sessions

\begin{tabular}{|c|c|c|c|c|c|c|}
\hline \multirow{2}{*}{ Measurement $\left(\mathrm{cm}^{3}\right)$} & \multirow{2}{*}{ First session } & \multirow{2}{*}{ Second session } & \multirow{2}{*}{ P-value } & \multirow{2}{*}{ Intrareader ICC } & \multicolumn{2}{|c|}{ Bland-Altman analysis } \\
\hline & & & & & Mean & $95 \%$ LOA \\
\hline Reader 1 & $26.1 \pm 40.0$ & $24.7 \pm 37.8$ & 0.795 & 0.995 (0.991 to 0.998) & 1.3 & 11.6 to -8.9 \\
\hline Reader 2 & $27.1 \pm 41.9$ & $29.6 \pm 45.0$ & 0.503 & 0.995 (0.988 to 0.997) & -2.5 & 9.0 to -14.0 \\
\hline Reader 3 & $25.8 \pm 38.6$ & $26.3 \pm 40.8$ & 0.924 & 0.996 (0.992 to 0.998) & -0.6 & 9.3 to -10.4 \\
\hline Reader 4 & $27.3 \pm 42.9$ & $25.1 \pm 36.8$ & 0.873 & 0.985 (0.971 to 0.992) & 2.3 & 21.3 to -16.7 \\
\hline P-value & 0.953 & 0.592 & & & & \\
\hline $\begin{array}{l}\text { Inter-reader } \\
\text { ICC }(95 \% \mathrm{Cl})\end{array}$ & $\begin{array}{c}0.996 \\
\text { (0.992 to 0.998) }\end{array}$ & $\begin{array}{c}0.994 \\
\text { (0.990 to 0.997) }\end{array}$ & & & & \\
\hline
\end{tabular}

Values are presented as mean \pm standard deviation unless otherwise indicated.

ICC, intraclass correlation coefficient; LOA, limits of agreement; Cl, confidence interval. 


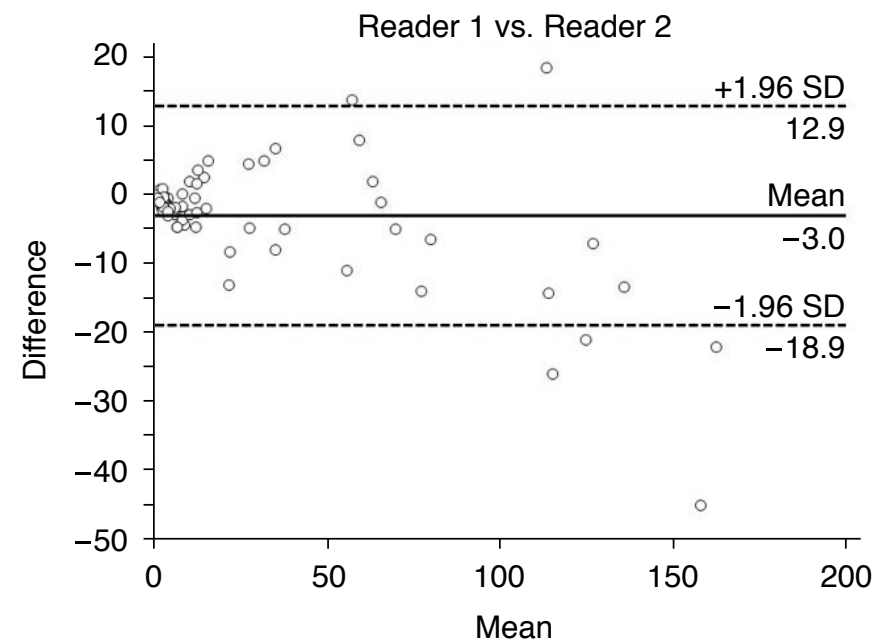

A

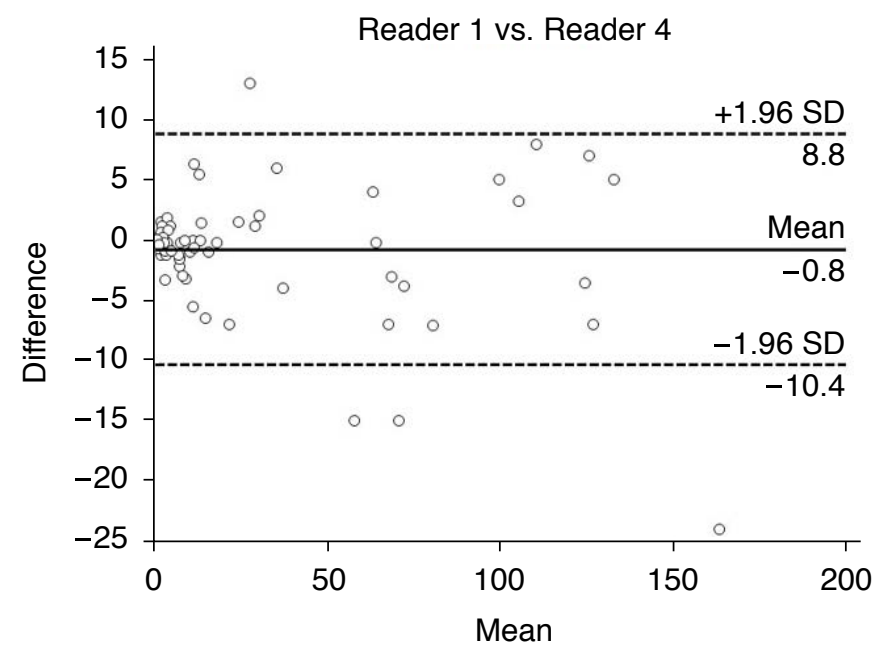

C

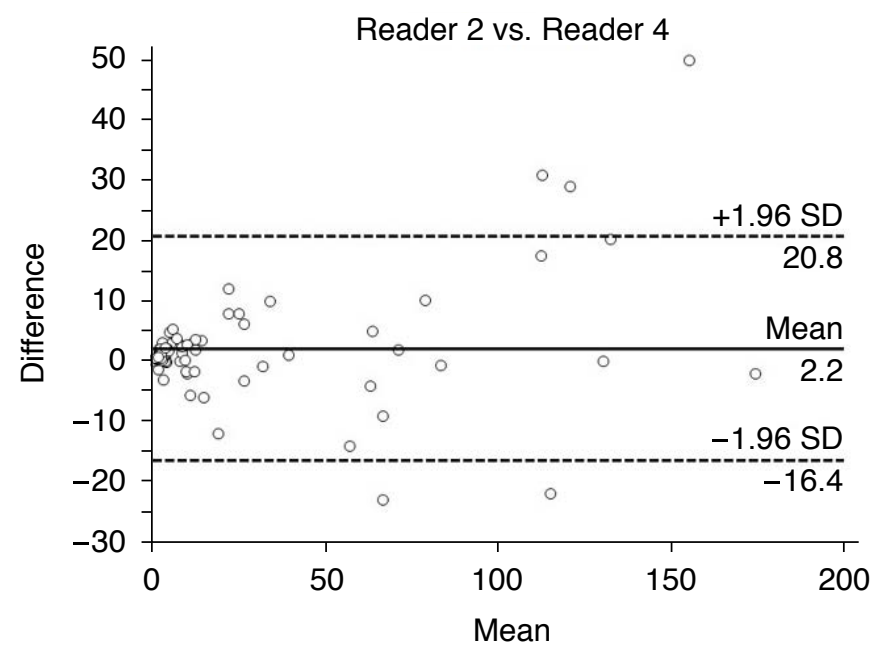

E

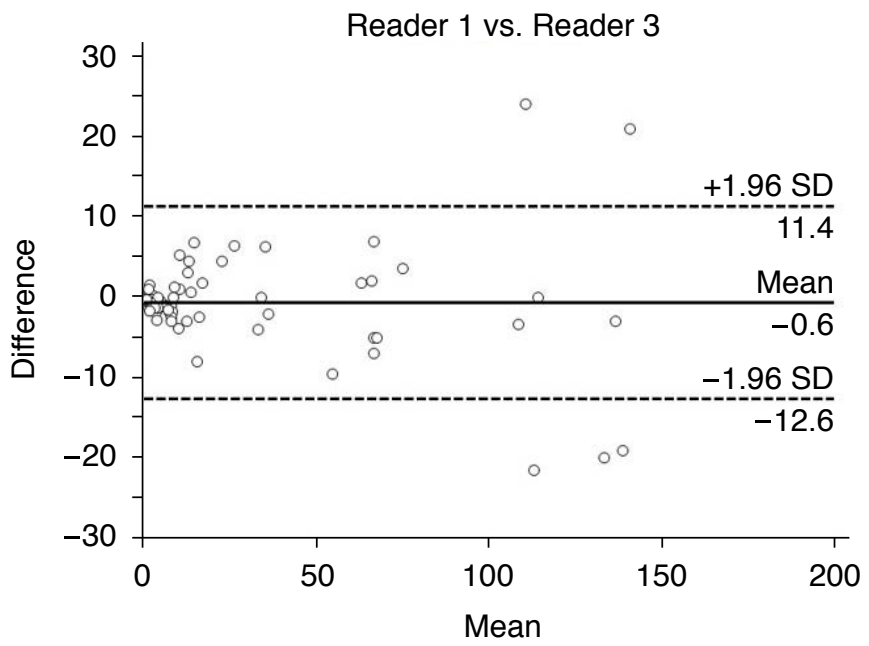

B

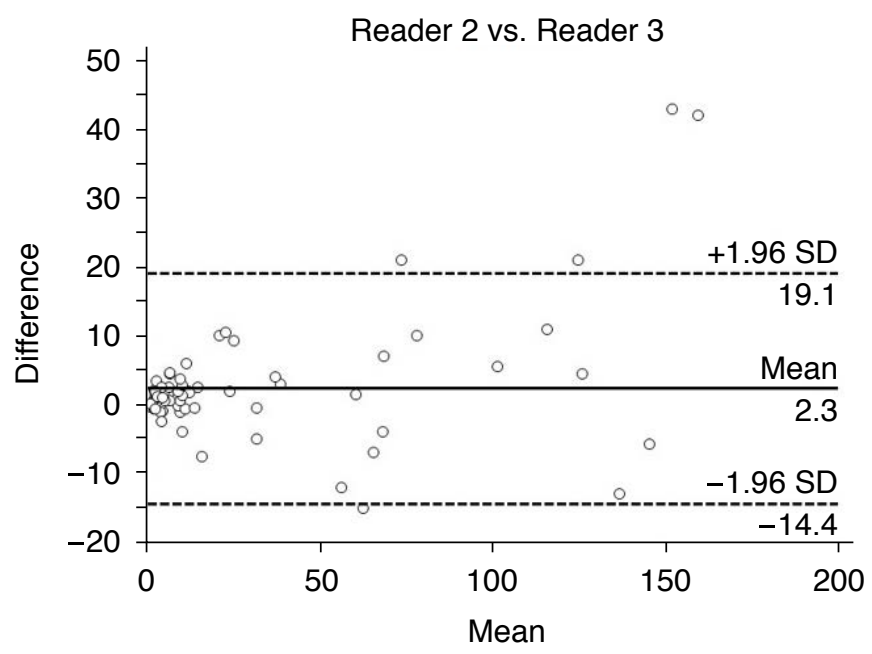

D

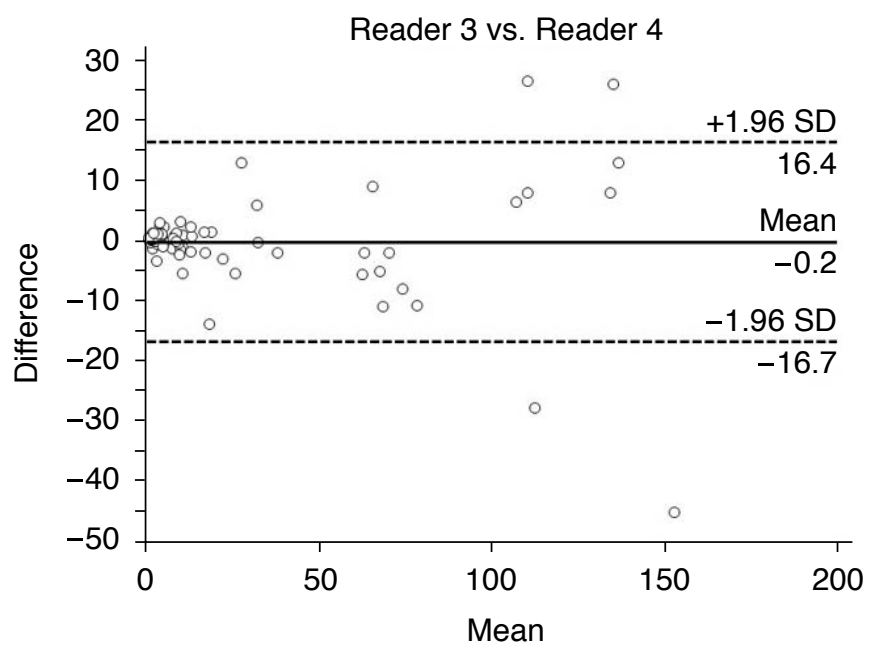

Fig. 1. Bland-Altman plot comparing inter-reader agreement $(A-F)$ for volume measurements of lesions. 


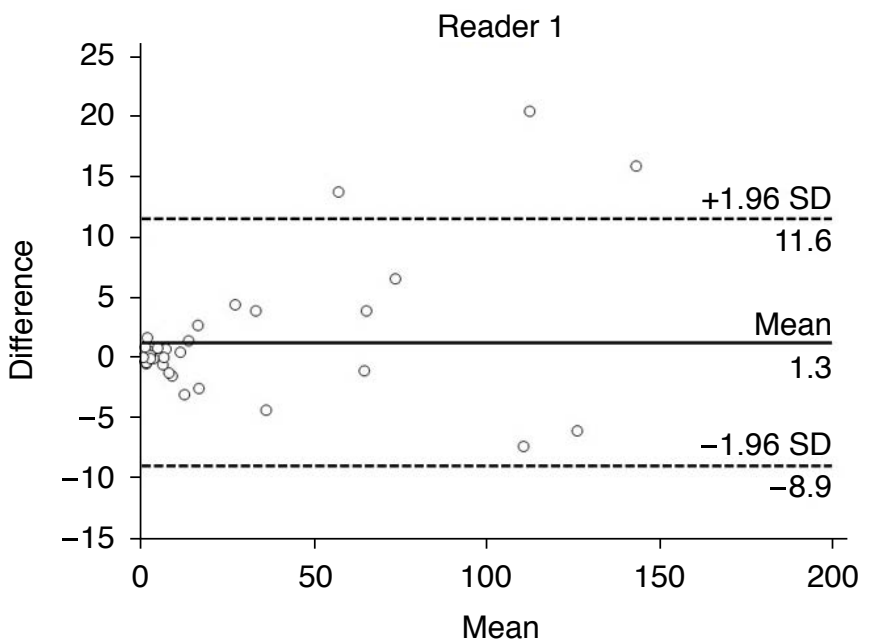

A

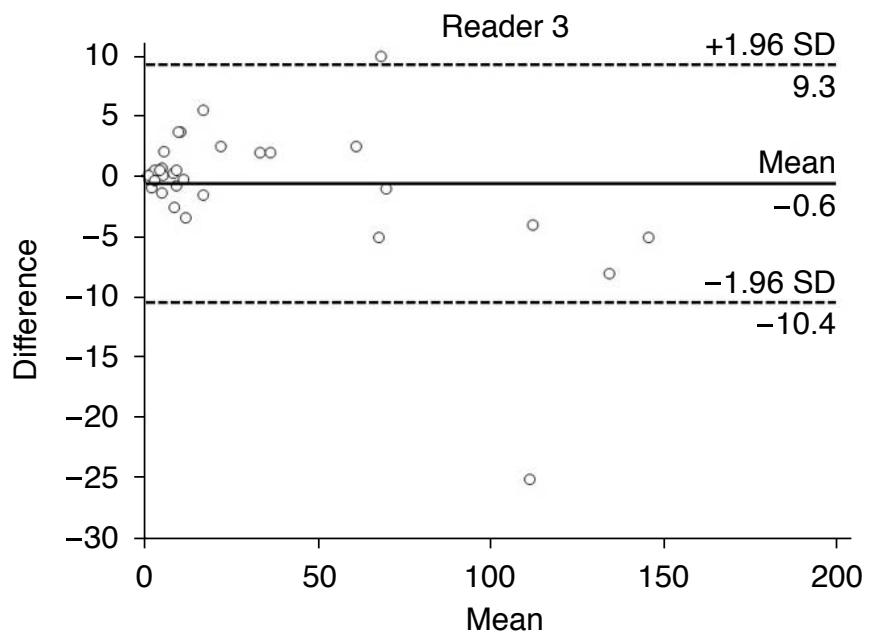

C

Fig. 2. Bland-Altman plot comparing intrareader agreement (A-D) for volume measurements of lesions.

Table 2. Volume measurements $\left(\mathrm{cm}^{3}\right)$ with inter-reader agreement according to the type of the focal liver lesion ( $\left.\mathrm{n}=39\right)$

\begin{tabular}{|c|c|c|c|c|c|c|c|c|c|c|}
\hline \multirow{2}{*}{ Lesion } & \multicolumn{2}{|c|}{ Reader 1} & \multicolumn{2}{|c|}{ Reader 2} & \multicolumn{2}{|c|}{ Reader 3} & \multicolumn{2}{|c|}{ Reader 4} & \multirow{2}{*}{$\begin{array}{c}\text { Mean } \\
\text { volume }\end{array}$} & \multirow{2}{*}{ ICC (95\% Cl) } \\
\hline & First & Second & First & Second & First & Second & First & Second & & \\
\hline $\mathrm{HCA}(n=1)$ & 151.0 & 153.0 & 173.0 & 180.0 & 130.0 & 138.0 & 175.0 & 130.0 & $151.5 \pm 21.4$ & NA \\
\hline $\mathrm{FNH}(\mathrm{n}=9)$ & $28.8 \pm 35.9$ & $29.3 \pm 38.4$ & $28.8 \pm 39.8$ & $32.0 \pm 43.9$ & $29.0 \pm 37.4$ & $28.5 \pm 38.9$ & $27.0 \pm 36.3$ & $27.3 \pm 35.4$ & $28.8 \pm 36.4$ & $0.998(0.996-1.000)$ \\
\hline $\begin{array}{l}\text { Hemangioma } \\
(n=6)\end{array}$ & $14.2 \pm 24.7$ & $12.3 \pm 19.2$ & $12.3 \pm 18.9$ & $15.3 \pm 23.2$ & $14.1 \pm 23.8$ & $14.4 \pm 22.7$ & $14.4 \pm 24.7$ & $15.0 \pm 25.0$ & $13.9 \pm 21.1$ & $0.998(0.995-1.000)$ \\
\hline $\mathrm{RN}(\mathrm{n}=1)$ & 1.1 & 1.3 & 2.9 & 1.6 & 1.6 & 2.1 & 1.3 & 1.6 & $1.8 \pm 0.7$ & NA \\
\hline $\mathrm{HCC}(n=6)$ & $23.6 \pm 28.3$ & $22.1 \pm 25.4$ & $26.0 \pm 30.8$ & $27.5 \pm 30.2$ & $25.8 \pm 25.8$ & $22.4 \pm 22.6$ & $28.2 \pm 29.7$ & $22.7 \pm 26.6$ & $24.7 \pm 25.5$ & $0.997(0.992-1.000)$ \\
\hline $\begin{array}{l}\text { RFA-treated } \\
\text { HCC }(n=9)\end{array}$ & $19.7 \pm 39.0$ & $17.4 \pm 32.3$ & $19.6 \pm 32.5$ & $17.0 \pm 30.9$ & $20.1 \pm 39.1$ & $19.9 \pm 39.1$ & $19.9 \pm 40.2$ & $18.2 \pm 30.6$ & $19.3 \pm 34.0$ & 0.997 (0.994-0.999) \\
\hline $\begin{array}{l}\text { Metastasis } \\
(n=7)\end{array}$ & $29.1 \pm 47.9$ & $29.1 \pm 49.6$ & $31.2 \pm 50.6$ & $31.5 \pm 52.4$ & $31.8 \pm 54.3$ & $33.0 \pm 56.6$ & $30.7 \pm 50.4$ & $30.3 \pm 49.3$ & $30.8 \pm 48.0$ & $0.999(0.997-1.000)$ \\
\hline
\end{tabular}

Values are presented as mean \pm standard deviation.

ICC, intraclass correlation coefficient; $\mathrm{Cl}$, confidence interval; HCA, hepatocellular adenoma; NA, not available due to the low number of cases; FNH, focal nodular hyperplasia; $\mathrm{RN}$, regenerative nodule; $\mathrm{HCC}$, hepatocellular carcinoma; RFA, radiofrequency ablation. 


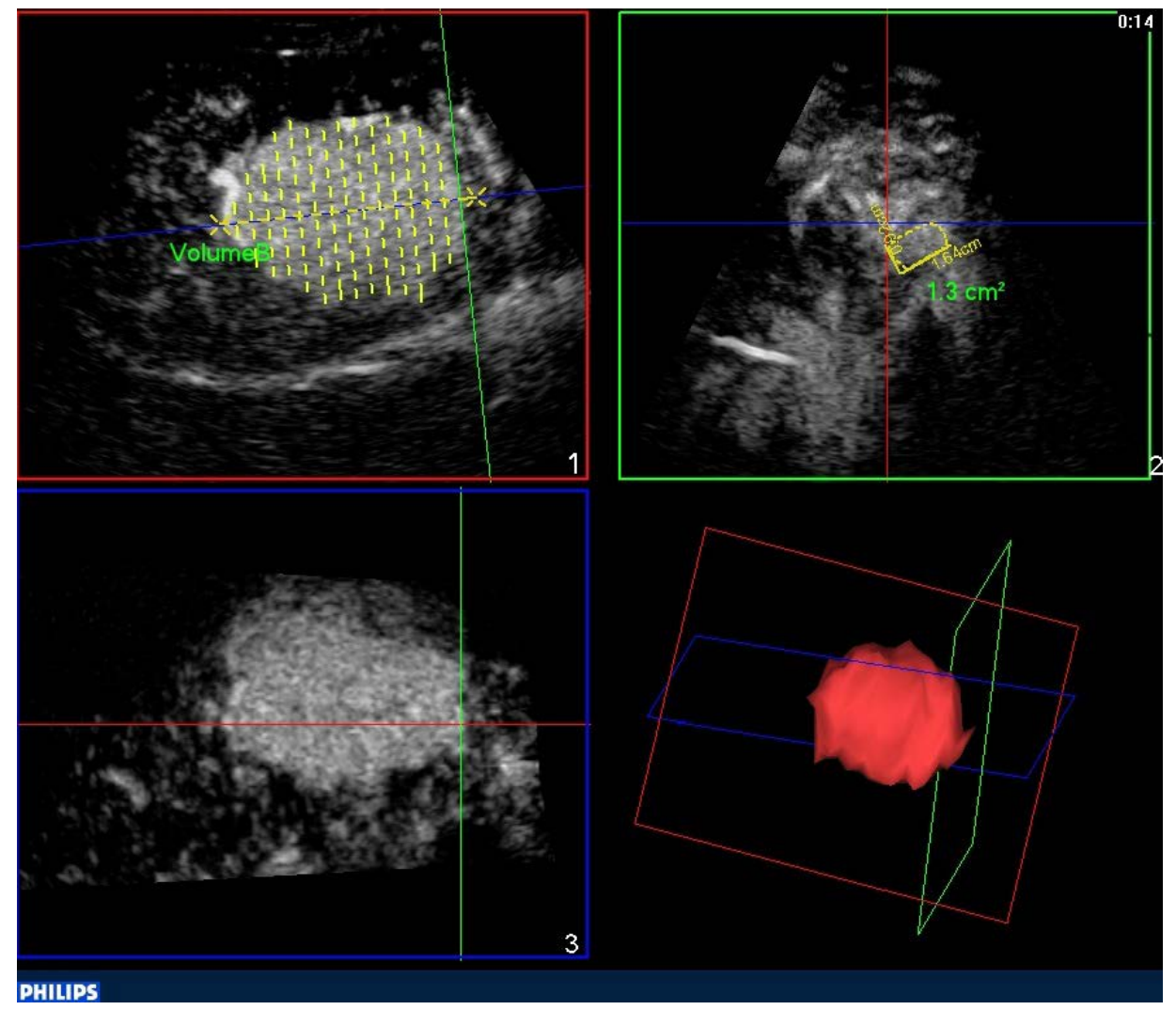

Fig. 3. Focal nodular hyperplasia in a 29-year-old woman (segment VIII). Three-dimensional contrast-enhanced ultrasound volumetric acquisition in the arterial phase (14 seconds after Sonovue injection) demonstrates a volume of $106.7 \mathrm{~cm}^{3}$.

Table 3. Intrareader agreement between the two reading sessions according to the type of focal liver lesions

\begin{tabular}{|c|c|c|c|c|}
\hline \multirow{2}{*}{ Lesion } & \multicolumn{4}{|c|}{ ICC (95\% Cl) } \\
\hline & Reader 1 & Reader 2 & Reader 3 & Reader 4 \\
\hline $\mathrm{HCA}(\mathrm{n}=1)$ & NA & NA & NA & NA \\
\hline FNH $(n=9)$ & $0.998(0.991-1.000)$ & $0.996(0.976-0.999)$ & $0.999(0.997-1.000)$ & $0.994(0.973-0.999)$ \\
\hline Hemangioma $(n=6)$ & $0.983(0.893-0.998)$ & $0.984(0.892-0.998)$ & $0.999(0.990-1.000)$ & $0.999(0.996-1.000)$ \\
\hline $\mathrm{RN}(\mathrm{n}=1)$ & NA & NA & NA & NA \\
\hline $\mathrm{HCC}(n=6)$ & $0.996(0.978-0.999)$ & $0.998(0.986-1.000)$ & $0.989(0.891-0.999)$ & $0.987(0.556-0.998)$ \\
\hline RFA-treated HCC $(n=9)$ & $0.990(0.961-0.998)$ & $0.987(0.946-0.997)$ & $0.985(0.940-0.997)$ & $0.976(0.896-0.995)$ \\
\hline Metastasis $(\mathrm{n}=7)$ & $0.999(0.995-1.000)$ & $0.994(0.964-0.999)$ & $0.999(0.997-1.000)$ & $0.998(0.989-1.000)$ \\
\hline
\end{tabular}

ICC, intraclass correlation coefficient; $\mathrm{Cl}$, confidence interval; HCA, hepatocellular adenoma; NA, not available due to the low number of cases; FNH, focal nodular hyperplasia; RN, regenerative nodule; HCC, hepatocellular carcinoma; RFA, radiofrequency ablation.

Table 4. Inter-reader agreement according to the postcontrast phase selected for volume measurements

\begin{tabular}{lcc}
\hline \multicolumn{1}{c}{ Postcontrast phase (number of lesions) } & First session ICC (95\% Cl) & Second session ICC (95\% Cl) \\
\hline Arterial $(n=17)$ & $0.995(0.991-0.998)$ & $0.993(0.984-0.997)$ \\
Portal venous $(n=9)$ & $0.998(0.996-1.000)$ & $0.997(0.993-0.999)$ \\
Late $(n=13)$ & $0.995(0.989-0.998)$ & $0.994(0.985-0.998)$ \\
\hline
\end{tabular}

Inter-reader agreement was assessed using intraclass correlation coefficients (ICCS), with 95\% confidence intervals (CIs). 
lesions, 3D-CEUS is readily available and does not rely on the use of ionizing radiation or potentially nephrotoxic contrast agents $[7,8,12]$. Furthermore, US is often the preferred imaging modality for guidance of loco-regional treatments, such as RFA [13]. In this setting, precise information about the shape and volume of a liver malignancy is crucial for optimizing tumor treatment in terms of type of needle electrode or the number of insertions $[14,15]$. Despite the potential importance of its role, the reproducibility of 3D-CEUS volume calculation still remains to be demonstrated. To our knowledge, this is the first study directly addressing the issue of the reproducibility of 3D-CEUS volume calculations.

In our study, the comparison of calculations of the volume of lesions among the four readers showed ICCS that were always above 0.994 and 0.985 for inter-reader and intrareader agreement, respectively. The practical interpretation of these results is almost perfect inter-reader agreement, without statistically significant differences between reading sessions. Interestingly enough, the readers involved in this study were not expert abdominal radiologists, but instead were radiology residents who had received only 5 hours of dedicated training. Hence, it might be hypothesized that, in the proper clinical setting, the specific process of volume calculation may be transferred to other professionals, thus sparing expensive medical time.

Our data showed almost perfect inter-reader agreement for all three postcontrast phases that were evaluated. This finding is of clinical relevance, since different postcontrast phases may provide useful information depending on the clinical scenario. The arterial phase is usually preferred for pre-treatment volume assessment of HCC or hypervascular metastases, whereas after loco-regional treatment of $\mathrm{HCC}$, volume measurements in the late or portalvenous phase may be helpful in order to ensure that the entire HCC lesion has been included in the treated volume $[9,14,15]$. This may explain why the four readers independently chose the same phase to assess for each lesion. After RFA, the finding that the volume of the ablation zone is greater than the volume of the lesion does not itself guarantee complete ablation. Hence, a more precise assessment of treatment efficacy by means of imaging techniques such as CEUS, $\mathrm{CT}$, or MRI is always needed. 3D-CEUS volume calculation might also allow an effective and early evaluation of antiangiogenic treatment of malignant tumors by encompassing the whole lesion and not only a section [16]. Of note, since shunts are not usually seen on CEUS, 3D-CEUS may allow for a more precise assessment of the true tumor volume in the arterial phase.

In our series we observed only one HCA. On CEUS, HCAs usually present a fast and centripetal arterial fill-in, followed by persistent enhancement in the extended portal-venous phase [17]. Nevertheless, the enhancement pattern may depend on the subtype of HCA, as well the presence of intralesional hemorrhage, necrosis, inflammation, and fat component [18]. The observed HCA was homogeneously, but slightly, hyperechoic in the arterial phase in comparison with the adjacent liver parenchyma. This may explain some discrepancy in our volume calculation. To a greater extent, if the FLL is isoechoic throughout the vascular phase, it may be difficult to obtain an accurate volume calculation.

Time consumption does not seem to represent a major limitation of the described 3D-CEUS technique, considering that in our experience a complete evaluation of each volumetric dataset lasted on average less than 2 minutes (mean, $118 \pm 25.5$ seconds). This is another inherent advantage of the semi-automated nature of the software used in this study, whereas manual measurements made by radiologists are quite time-consuming.

This prospective study had some limitations. First, the patient population was relatively small. Second, we only included lesions suitable for 3D-CEUS, which may have led to selection bias, especially regarding deeply located lesions, which often are less suitable for CEUS. Nevertheless, this limitation did not affect the reproducibility assessment, at least when considering satisfactory 3D-CEUS examinations. Furthermore, in our study an intermittent imaging modality was used, whereas the introduction of novel technologies, such as new electronic matrix probes, allows real-time 3D volumetric evaluation of FLLs during the entire vascular phase [6].

In conclusion, 3D-CEUS assisted by semi-automatic software provided a fast and reproducible method to calculate the volume of FLLs, and this technique might be useful as an alternative to CT for pre- and post-treatment assessment of liver tumors.

ORCID: Tommaso Vincenzo Bartolotta: https://orcid.org/0000-0002-8808-379X; Roberto Cannella: https://orcid.org/0000-0002-3808-0785; Giorgia Porrello: https:// orcid.org/0000-0003-1617-5609; Rossana Taravella: https://orcid.org/0000-00028253-3294; Adele Taibbi: https://orcid.org/0000-0001-6442-744X

\section{Author Contributions}

Conceptualization: Bartolotta TV, Taibbi A. Data acquisition: Sidoti Pinto A, Porrello G, Taravella R, Randazzo A. Data analysis or interpretation: Bartolotta TV, Cannella R. Drafting of the manuscript: Bartolotta TV. Critical revision of the manuscript: Bartolotta TV, Cannella R, Taibbi A. Approval of the final version of the manuscript: all authors.

\section{Conflict of Interest}

Tommaso Vincenzo Bartolotta serves as Editor for the Ultrasonography, but has no role in the decision to publish this article. All remaining authors have declared no conflicts of interest. 


\section{References}

1. Ferraioli G, Meloni MF. Contrast-enhanced ultrasonography of the liver using SonoVue. Ultrasonography 2018;37:25-35.

2. Bartolotta TV, Taibbi A, Midiri M, Lagalla R. Contrast-enhanced ultrasound of hepatocellular carcinoma: where do we stand? Ultrasonography 2019;38:200-214.

3. Bartolotta TV, Taibbi A, Picone D, Anastasi A, Midiri M, Lagalla R. Detection of liver metastases in cancer patients with geographic fatty infiltration of the liver: the added value of contrast-enhanced sonography. Ultrasonography 2017;36:160-169.

4. Dong FJ, Xu JF, Du D, Jiao Y, Zhang L, Li M, et al. 3D analysis is superior to $2 \mathrm{D}$ analysis for contrast-enhanced ultrasound in revealing vascularity in focal liver lesions: a retrospective analysis of 83 cases. Ultrasonics 2016;70:221-226.

5. Luo W, Numata K, Morimoto M, Kondo M, Takebayashi S, Okada $M$, et al. Focal liver tumors: characterization with 3D perflubutane microbubble contrast agent-enhanced US versus 3D contrastenhanced multidetector CT. Radiology 2009;251:287-295.

6. Cao J, Dong Y, Fan P, Mao F, Wang W. Feasibility of dynamic threedimensional contrast-enhanced ultrasound in focal liver lesions: image quality evaluation and correlation of quantification with two-dimensional contrast-enhanced ultrasound. Clin Hemorheol Microcirc 2019;72:305-316.

7. Yan J, Schwartz $L H$, Zhao B. Semiautomatic segmentation of liver metastases on volumetric CT images. Med Phys 2015;42:62836293.

8. Yamamichi J, Kawaguchi Y, Otsuka T, Nakashita S, Mizobe H, Eguchi $Y$, et al. Assessment of tumor volume and density as a measure of the response of advanced hepatocellular carcinoma to sorafenib: application of automated measurements on computed tomography scans. JGH Open 2020;4:145-152.

9. Bartolotta TV, Taibbi A, Matranga D, Midiri M, Lagalla R. 3D versus $2 D$ contrast-enhanced sonography in the evaluation of therapeutic response of hepatocellular carcinoma after locoregional therapies: preliminary findings. Radiol Med 2015;120:695-704.

10. Song Y, Cheng J, Zhang R. Contribution of 3-dimensional contrastenhanced ultrasonography (CEUS) compared with 2-dimensional CEUS in the analysis of liver tumors. J Ultrasound Med 2018;37:1117-1128.

11. Downey DB, Fenster A, Williams JC. Clinical utility of threedimensional US. Radiographics 2000;20:559-571.

12. Keil S, Behrendt FF, Stanzel S, Suhling M, Koch A, Bubenzer J, et al. Semi-automated measurement of hyperdense, hypodense and heterogeneous hepatic metastasis on standard MDCT slices. Comparison of semi-automated and manual measurement of RECIST and WHO criteria. Eur Radiol 2008;18:2456-2465.

13. Dodd GD 3rd, Soulen MC, Kane RA, Livraghi T, Lees WR, Yamashita Y, et al. Minimally invasive treatment of malignant hepatic tumors: at the threshold of a major breakthrough. Radiographics 2000;20:927.

14. Cao J, Dong Y, Mao F, Wang W. Dynamic three-dimensional contrast-enhanced ultrasound to predict therapeutic response of radiofrequency ablation in hepatocellular carcinoma: preliminary findings. Biomed Res Int 2018;2018:6469703.

15. Bartolotta TV, Taibbi A, Midiri M, De Maria M. Hepatocellular cancer response to radiofrequency tumor ablation: contrast-enhanced ultrasound. Abdom Imaging 2008;33:501-511.

16. Lassau N, Chebil M, Chami L, Bidault S, Girard E, Roche A. Dynamic contrast-enhanced ultrasonography (DCE-US): a new tool for the early evaluation of antiangiogenic treatment. Target Oncol 2010;5:53-58.

17. Bartolotta TV, Vernuccio F, Taibbi A, Lagalla R. Contrast-enhanced ultrasound in focal liver lesions: where do we stand? Semin Ultrasound CT MR 2016;37:573-586.

18. Manichon AF, Bancel B, Durieux-Millon M, Ducerf C, Mabrut JY, Lepogam MA, et al. Hepatocellular adenoma: evaluation with contrast-enhanced ultrasound and MRI and correlation with pathologic and phenotypic classification in 26 lesions. HPB Surg 2012;2012:418745. 